\title{
Factors Affecting Use of Telemedicine and Telesurgery in Cancer Care (TTCC) among Specialist Physicians
}

\author{
Mohammadreza Maleki ${ }^{1}$, Seyed Masood Mousavi ${ }^{1 *}$, Omid Khosravizadeh ${ }^{2}$, \\ Mohammad Heidari ${ }^{3}$, Mehdi Raadabadi ${ }^{4}$, Mina Jahanpour ${ }^{5}$
}

\begin{abstract}
Background: Considering the increasing incidence of different cancers, use of modern technologies such as TTCC can make a dramatic change in treatment of these diseases. So, if the evolution strategy conflicts with the organizational culture, national culture, and organizational structure of experts in this field, resistance will rise. Objective: The purpose of this study was to determine the effective factors on the use of TTCC in hospitals affiliated to Tehran University of Medical Sciences using the AHP model. Materials and Methods: This current descriptive study was carried out in 2018 on specialist physicians from five hospitals of Tehran University of Medical Sciences. The data gathering tool was a questionnaire consisting of two sections; demographic items and 27 effective factors influencing the implementation of TTCC technology, which was evaluated for validity and reliability. Data analysis was performed using analytical hierarchy process with expert choice and for empirical illustration are used to discuss the use of covariance-based SEM versus smart PLS software. Results: Based on AHP comparisons in the studied factors, the highest priority was the Cultural factor. In other words, commitment of senior executives to support the provision of a new technology with a weight of 0.327 . The lowest priority, however, was related to support diverse approaches, innovation, creativeness, and acceptance of new ideas with a weight of 0.038 . In regard with technical and organizational factors, out-of-hospital access to the intranet network and support provided by the doctors, with the weight of 0.221 and 0.205 in order, acquired the highest rates. Conclusion: Considering the high prevalence of cancer in Iran and the necessity of using new technologies in its treatment and by addressing the specialists' views and opinions in this field, organizational and national culture in the application of TTCC technology should be promoted. This target can be hit through acceptance, change in attitude, and successful use of TTCC technology by medical professionals.
\end{abstract}

Keywords: Teletherapy- telesurgery- analytical hierarchy process (AHP)

Asian Pac J Cancer Prev, 19 (11), 3123-3129

\section{Introduction}

The term "teletherapy" means distance communication. From the view point of information technology, it means providing medical services from a distant distance and through networks that support transmission of sound, video, and computer data. Telemedicine or electronic health means health care as well as methods of prevention, diagnosis, and treatment of diseases through electronic and communicative processes (Edirippuliges et al, 2009). Further, telesurgery is widely defined as the ability to perform surgeries from long distances using modern surgical techniques and by overcoming the obstacles of time and distance (Pande et al., 2003). During this procedure, the surgeon does not have physical presence in the place where the patient or model is operated (Prokosch et al., 2006). In this surgical procedure, the surgeon does not have physical presence to visit the patient, so, the observation and manipulation of the surgical site is performed through electronic equipment (Stanberry, 2000). The ultimate goal of telesurgery is to enable the specialist surgeon to be present virtually on the patient's bedside due to reasons like distance (for example, in remote and rural areas), special conditions (such as a battlefield or accident scene), risks that may be caused by patients for the surgical team (such as infectious diseases and radioactive contamination), or the risks posed by the surgical team that threaten patient's health (e.g., immune deficiency in a patient). Telemedicine includes extensive services such as distance counseling, teleradiology, and telesurgery (Kumar, 2008).

${ }^{1}$ Health Services Management Department, School of Health Management and Information Sciences, Iran University of Medical Sciences, ${ }^{4}$ Students Scientific Research Center, Tehran University of Medical Sciences, ${ }^{5}$ Social Welfare Management Research Center, University of Social Welfare and Rehabilitation Sciences, Tehran, ${ }^{2}$ Social Determinants of Health Research Center, Qazvin University of Medical Sciences, Qazvin, ${ }^{3}$ Department of Medical and Surgical, School of Nursing and Midwifery, Shahrekord University of Medical Sciences, Shahrekord, Iran. *For Correspondence: m.mousavihp93@gmail.com 
Provision of telemedicine services actually contains very broad concepts from the viewpoints of communication technology and medical services. The breadth of these concepts is so that we can call telemedicine a culture in applying the features of communication concepts regarding provision of health services than a service. This broadness, in its simplest possible form, ranges from a simple telephone connection for a psychotherapy service, to applying an intelligent agent in an intercontinental relationship, using vital signs and various medical images for diagnosis and treatment of a patient (Oh et al., 2005). Technology issues of telemedicine, distance learning, selection of a long distance communication platform, as well as voice and video technology are a network of telemedicine infrastructures. Saving time, reducing the cost of several visits to the office, using the patients' database to examine the process of disease recovery, using the experiences of physicians and specialists from other parts of the world, improving the provision of medical services to rural and remote areas, increasing access to health care services, improving the quality of health care services, reducing medical mistakes, and exchanging new medical findings among physicians around the world are from the benefits of telemedicine. However, as with new technologies, there are difficulties in providing the necessary conditions to make the telemedicine applications public so that everyone can have access to them. Barriers such as lack of familiarity with telemedicine technology, need for sophisticated technical and electronic infrastructure, and the high cost of equipment are some examples. Moreover, maintaining information security need legal methods to track mistakes and negligence in telemedicine (Hung and Zhang, 2003).

Cancer, after cardiovascular diseases, is the second most common cause of death in developed states and the third one in less developed countries (Shahbazi and Heidari, 2014; Mahoozi et al., 2017; Raeissi et al., 2017; Shahbazi et al., 2017). It is also estimated to be a major contributor to global disease burden in the coming decades. The number of new cases is expected to increase to 17 million people in 2020 (Adami et al., 2008; Youlden et al., 2012). Studies showed that both physicians and patients were very satisfied with the use of technology in treatment of diseases (Wysocki et al., 2005; Hazin and Qaddoumi, 2010; Hede, 2010).

Considering various above-mentioned factors involved in the TTCC infrastructure, it is necessary to develop a comprehensive model for identifying and prioritizing these factors. In order to achieve this, a proper decision model is needed to make choices in situations where decision-making criteria are different and sometimes conflicting. One of the effective ways is to use the Analytical Hierarchy Process (AHP) (Leung and Cao, 2000). In this method, the decision maker begins by providing a hierarchical tree that shows the indicators and decision options. The decision maker then performs a set of paired comparisons which indicate the weight of each factor compared with the rival options. Finally, the AHP logic integrates the matrices derived from the paired comparisons into the optimal decision making. Furthermore, the consensus nature of the group decision-making improves the compatibility of judgments and upgrades the model's reliability as a decision-making tool (Azar and Faraji, 2010). The biggest advantage of this method is reduction of comparisons' incompatibility. Therefore, the inconsistency rate shows how much confidence can be gained from the comparative priorities. In this regard, the purpose of this study was to determine the effective factors on the use of TTCC in hospitals affiliated to Tehran University of Medical Sciences using the AHP model.

\section{Materials and Methods}

\section{Setting}

The current descriptive study was conducted in 2017. The research environment was five hospitals selected from the hospitals affiliated to Tehran University of Medical Sciences in which the TTCC implementation conditions were more powerful. The hospitals were Heart Center, Farabi, Sina, Imam, and Amir Aalam. Since hospitals with more powerful TTCC implementation conditions were required, a non-random sampling was used. These hospitals were then selected by two professors who manage the hospitals at the university. In some cases, these people were also among the hospital observers who had the necessary knowledge about the hospitals. Thus, these informed professionals selected five hospitals with the required conditions for implementation of telemedicine in which conduction of a study was more effective than other hospitals. In the studied hospitals, all physicians and specialists working as faculty members were investigated (96 people); therefore, no sampling was conducted.

\section{Ethical approval}

After selecting the eligible participant, the researcher was introduced to them and the objectives of the study were elaborated for the participants. The informed consent was obtained from the subjects and they were assured that their information will remain confidential.

\section{Data Collection}

The existing questionnaire only includes the factors affecting use of TTCC in the treatment of cancer and eventually reports the frequency of these factors. So, in this study, among all the effective factors, those with a greater role in the implementation culture were selected according to the experts' opinions. These factors were later organized in the form of a researcher-made questionnaire to be prioritized by participants. This questionnaire consisted of two parts. The first part included demographic questions such as age, gender, being a faculty member or not, work experience, academic degree, education, and marital status. The second part of the questionnaire included 3 dimensions such as, cultural, technical and organizational, each dimension contain 9 items. In Table 1 all items were listed.

The purpose was to compare and determine significance of the studied criteria from the viewpoint of expert physicians. Thus, questions were designed so that each factor was compared with other factors based on the numbers, $9,7,5,3,1$, representing concepts of 
completely more important, more important, important, a bit important, and identical. Based on this, the first factor was compared with the next eight factors, the second factor was compared with the next seven factors, and the eighth factor was compared with the ninth one, making a total number of 36 comparisons. The selection of factors was carried out by reviewing the texts and articles in this field and interviewing with the knowledgeable experts. After designing, the validity of the questionnaire was evaluated through the expert opinions of three faculty members. Its reliability was then confirmed by calculating Cronbach alpha value $(\alpha=0.88)$. The questionnaires were distributed among the physicians after making coordination with the hospital's head. After participants were provided with a brief explanation about the research, they were asked to complete the questionnaires.

\section{Statistical analysis}

The collected data were introduced into Excel 2010 software. Data analysis was performed using analytical hierarchy process with expert choice and for empirical illustration are used to discuss the use of covariance-based SEM versus PLS path modeling. PLS path modeling is suited for analyzing exploratory models with no rigorous theory grounding g. Except for the four structure models derived from AHP method, there is likely exiting some
Telemedicine and Telesurgery in Cancer Care

relationship be- tween latent variables that To examine the fit of the structural model of the research, several criteria are used, the first and most fundamental criterion is the $\mathrm{Z}$ coefficients. The fitting of a structural model using coefficients $T$ is such that these coefficients should be greater than 1.96 to be confirmed at $95 \%$ confidence level (Chiang, 2013).

\section{Results}

With the weight of 3.90 , access of clinical and non-clinical units to the Internet turned out to have the highest mean among technical factors associated with the implementation of remote surgery; with a mean of 4.13, this position; i.e. the highest mean, was occupied by the support provided by the doctors among organizational doctors. In regard with cultural factors, accepting the objectives of the organization and getting involved in fulfilling these objectives on the part of doctors and the other staff turned out to be of the highest mean; i.e. 3.81.

From the 96 physicians studied in this research, majority were from 31 to 40 years old. In terms of gender, most specialists were male. Most of participants were officially employed and worked in the field of education. They were mostly assistant professors in terms of scientific degree. Regarding the marital status, majority of them

Table 1. The Mean Scores Technical, Organizational and Cultural Factors Affecting the Implementation of Remote Surgical Technology

\begin{tabular}{|c|c|c|c|c|c|}
\hline $\mathrm{SD}$ & Mean & SD & Mean & Component and Variables examined & \\
\hline \multirow{9}{*}{1.10} & \multirow{9}{*}{3.52} & 1.29 & 3.58 & Integrity in providing and distributing information & \multirow{9}{*}{$\begin{array}{l}\text { Cultural } \\
\alpha=0.92\end{array}$} \\
\hline & & 1.23 & 3.75 & Performing continuous or in-service training programs & \\
\hline & & 1.10 & 3.80 & The commitment of senior executives to support the provision of new technology & \\
\hline & & 1.39 & 3.67 & Free expression of mistakes and successes & \\
\hline & & 1.51 & 3.35 & The existence of clear rules and regulations without any ambiguity & \\
\hline & & 1.50 & 3.25 & Implementation of leadership practices and policies in the organization & \\
\hline & & 1.64 & 3.12 & Supporting diverse approaches, creativeness, innovations, and acceptance of new ideas & \\
\hline & & 1.45 & 3.81 & Participation and acceptance of the organization goals by doctors and staffs & \\
\hline & & 1.36 & 3.42 & The existence of organizational communication between high and low ranks of the organization & \\
\hline \multirow{9}{*}{1.31} & \multirow{9}{*}{3.34} & 1.36 & 3.90 & Access to all clinical and non- clinical units on the internet & \multirow{9}{*}{$\begin{array}{c}\text { Technical } \\
\alpha=0.91\end{array}$} \\
\hline & & 1.36 & 3.35 & Ability to communicate between different systems & \\
\hline & & 1.37 & 3.40 & out-of-hospital access to the intranet network & \\
\hline & & 1.17 & 3.77 & Use video conferencing & \\
\hline & & 1.26 & 3.83 & Use uppercase and lowercase letters to encrypt & \\
\hline & & 1.35 & 2.97 & Equipped with optical fiber & \\
\hline & & 1.32 & 3.02 & Equipped with copper cable & \\
\hline & & 1.38 & 2.92 & Equipped with small electromagnetic waves & \\
\hline & & 1.45 & 2.92 & Equipped with high resolution monitors & \\
\hline \multirow{9}{*}{1.02} & & 1.27 & 3.57 & The existence of a strategic telemedicine implementation plan & \multirow{9}{*}{$\begin{array}{c}\text { Organizational } \\
\quad \alpha=0.94\end{array}$} \\
\hline & & 1.31 & 3.47 & Structural changes for remote medical reception & \\
\hline & & 1.34 & 3.27 & Having a curriculum in medical development & \\
\hline & & 1.34 & 3.38 & The prospect of the deployment of telemedicine & \\
\hline & 3.51 & 1.28 & 3.72 & Positive viewpoints for the establishment of telemedicine & \\
\hline & & 1.36 & 3.18 & University level information & \\
\hline & & 1.38 & 3.42 & Private sector capability & \\
\hline & & 1.19 & 3.50 & Community support for the development of telemedicine & \\
\hline & & 1.02 & 4.13 & support provided by the doctors & \\
\hline
\end{tabular}


Table 2. Prioritization of Effective Factors on Using TTCC

\begin{tabular}{|c|c|c|c|}
\hline Dimensions & Factors & Symbol & Indicator's weight \\
\hline \multirow{8}{*}{ Cultural } & Integrity in providing and distributing information & Q 1 & 0.039 \\
\hline & Performing continuous or in-service training programs & Q 2 & 0.077 \\
\hline & The commitment of senior executives to support the provision of new technology & Q 3 & 0.327 \\
\hline & Free expression of mistakes and successes & Q 4 & 0.040 \\
\hline & The existence of clear rules and regulations without any ambiguity & Q 5 & 0.068 \\
\hline & Implementation of leadership practices and policies in the organization & Q 6 & 0.077 \\
\hline & Supporting diverse approaches, creativeness, innovations, and acceptance of new ideas & Q 7 & 0.038 \\
\hline & Participation and acceptance of the organization goals by doctors and staffs & Q 8 & 0.262 \\
\hline \multirow{10}{*}{ Technical } & The existence of organizational communication between high and low ranks of the organization & Q 9 & 0.071 \\
\hline & Acceess to all clinical and non- clinical units on the internet & Q 10 & 0.123 \\
\hline & Ability to communicate between different systems & Q 11 & 0.13 \\
\hline & out-of-hospital access to the intranet network & Q 12 & 0.221 \\
\hline & Use video conferencing & Q 13 & 0.198 \\
\hline & Use uppercase and lowercase letters to encrypt & Q 14 & 0.063 \\
\hline & Equipped with optical fiber & Q 15 & 0.068 \\
\hline & Equipped with copper cable & Q 16 & 0.068 \\
\hline & Equipped with small electromagnetic waves & Q 17 & 0.084 \\
\hline & Equipped with high resolution monitors & Q 18 & 0.045 \\
\hline \multirow{9}{*}{ Organizational } & The existence of a strategic telemedicine implementation plan & Q 19 & 0.109 \\
\hline & Structural changes for remote medical reception & Q 20 & 0.067 \\
\hline & Having a curriculum in medical development & Q 21 & 0.162 \\
\hline & The prospect of the deployment of telemedicine & Q 22 & 0.089 \\
\hline & Positive viewpoints for the establishment of telemedicine & Q 23 & 0.119 \\
\hline & University level information & Q 24 & 0.055 \\
\hline & Private sector capability & Q 25 & 0.128 \\
\hline & Community support for the development of telemedicine & Q 26 & 0.066 \\
\hline & support provided by the doctors & Q 27 & 0.205 \\
\hline
\end{tabular}

were married. Based on hierarchical analysis and paired comparisons of the investigated factors, the highest weight or priority was attributed to the commitment of senior executives to support the provision of a new technology with a weight of 0.327 . The lowest weight or priority, however, was related to supporting diverse approaches, innovation, creativeness, and acceptance of new ideas with a weight of 0.038 (Table 2).
In regard with technical and organizational factors, out-of-hospital access to the intranet network and support provided by the doctors, with the weight of 0.221 and 0.205 in order, acquired the highest rates.

The inconsistency rate for these indicators was 0.06 . Given that the inconsistency rate should be less than 0.1 and this value was less than 0.10 for factors, the calculated weights and priorities are acceptable and there is an

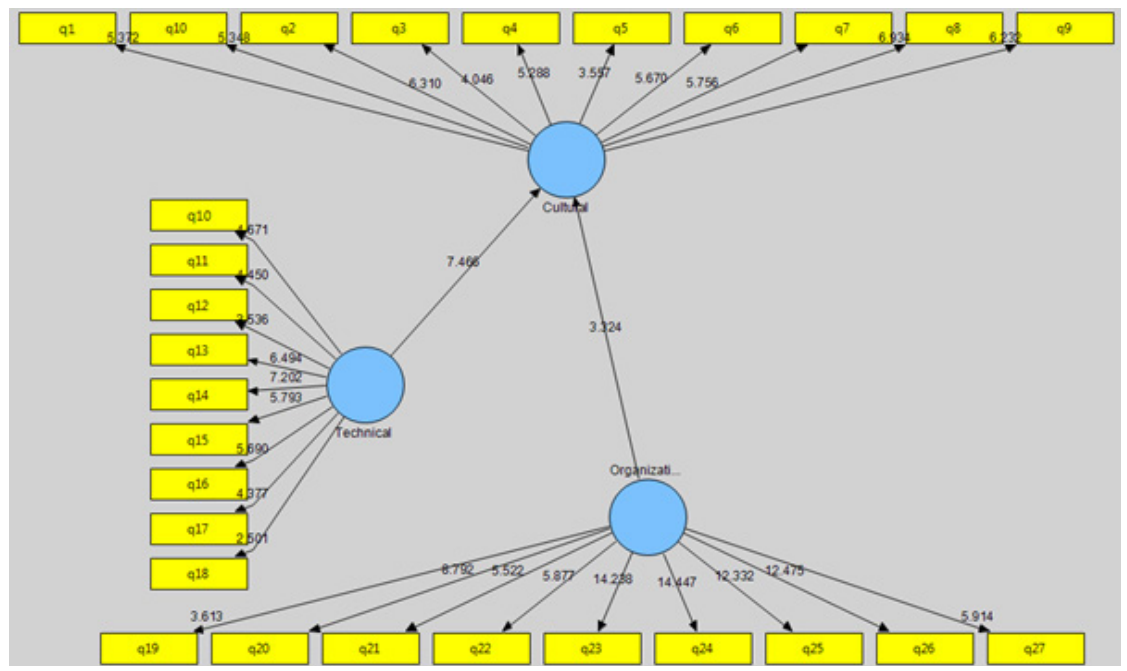

Figure 1. Significant Coefficients Z (Values of T-Value) 
acceptable consistency among the samples' viewpoints.

Several factors, the first and most fundamental of which was $\mathrm{Z}$ coefficients, were used to assess the fitness of the structural model of the research. As indicated in the above Figure, it can be stated that paths with (T) coefficient higher than 1.96 are showed significance and suitability with a confidence level of $0.95 \%$.

\section{Discussion}

Current developments in telemedicine technology have brought about a significant transformation in the majority of aspects of human life; these changes have had a significant impact on human life and the delivery of services; they have, also, created new needs which necessitates novel services in this area (Rho et al., 2015). The present study was conducted to analyze technical, organizational and cultural factors effective on the establishment of TTCC in hospitals from the viewpoint of specialized physicians. According to the results presented in Table 2, it can be concluded that, as consistent with several formerly conducted studies, managers' paying attention to the development of Internet infrastructure and access to all clinical and non-clinical units have acquired the highest means.

According to the results of the present research, cultural infrastructure for the implementation of remote surgery was one of the most noticeable strengths of the implementation of remote medical technology services in the hospitals. Therefore, it seems logical to claim that one of the most important obstacles to the implementation of remote medicine; i.e. resistance on the part of physicians, care providers and patients, has been, somehow, resolved in under study patients. In a study conducted in 2015, entitled "Factors effective on the efficient use of telemedicine in hospitals", Merchant et al., (2015) emphasized the importance of organizational culture as an inseparable a necessity for the successful implementation of telemedicine.

The results of a 2014 study in the United States, quick response to health care issues, monitoring patients with chronic diseases such as cancer, improving the access of patients living in remote areas, compensating for career shortages of experienced professionals, decreasing the costs and quick response by doctors to emergencies, all of which need to be strengthened by technical, organizational and cultural factors, have been introduced as the most important and prioritized reasons for using telemedicine in healthcare industry, a point which turned out to be quite consistent with the findings of the present research (Weinstein et al., 2014).

Based on hierarchical analysis and paired comparisons in the examined factors, the highest priority was related to senior executives' commitment to support the provision of a new technology with a weight of 0.327 . The lowest weight or priority, however, was associated with supporting diverse approaches, creativeness, innovation, and acceptance of new ideas with a weight of 0.038 .

From the studies conducted on the factors affecting experts' use of TTCC, it follows that the culture and organizational structure have a positive role in the successful operation and implementation of TTCC technology in hospitals and other healthcare providing centers (Field, 1996). Hossein et al., (2005) they investigated 82 faculty members and reported the success factors of telemedicine as the following: senior executives' commitment to support telemedicine technology $(95 \%$,), implementation of continuous training programs for doctors and employees (94\%), implementation of leadership practices and policies $(78.8 \%)$, honesty in providing and distributing information (85.3\%), supporting diverse approaches, innovation, and acceptance of new ideas by managers $(81.7 \%)$, determining clear and strategic perspectives and plans $(78 \%)$, participation and acceptance of organizational goals by physicians and staffs (77\%), clear and unambiguous organizational rules and regulations (68\%), the existence of bilateral organizational communications from top to bottom, and vice versa $(62.2 \%)$.

Janet et al., (2005) introduced written policies as a tool for organizational readiness in telemedicine. Also emphasized on the importance of various aspects of work planning and preparedness (preparing the required staff, providing the telemedicine coordinator, and technical readiness) in the success of telemedicine in another study. Janet et al., (2003) argued that organizational readiness is a multidimensional concept related to planning and working environment. The role of active leadership of the organization and membership of the leader in the telemedicine team is also of great importance (Patterson, 2005). Supporting physician acts as the organization's attorney, plays the leading role in collecting physicians and user groups' views, and assures that doctors had sufficient inputs in the decision-making process (Mastaneh et al, 2010). Other researches were also conducted on the organizational aspects involved in the success and implementation of telemedicine, which mainly focused on organizational characteristics and activities such as tendency to learn, avoidance of uncertainty (Bangert and Doktor, 2003), successful marketing of telemedicine systems, and availability of appropriate information systems within the organization (Welsh, 2002). The main point from various researchers' viewpoint is the importance of having a written and formal agenda for optimal establishment of telemedicine system (Najafi et al., 2007). Rahimzadeh et al., (2013) investigated the feasibility of establishment and deployment of telemedicine in Imam Khomeini Hospital of Ardebil. He stated that the principle of organizational culture is appropriate for establishment of telemedicine and reported that this factor gained a higher score than other studied components in the research. Further noted that this desirable organizational culture for establishment of telemedicine is the result of hospital managers' high commitment towards educational and executive activities. In the same vein, Bangert and Doktor (2000) studied the role of organizational culture in management of telemedicine systems in 2013 and found that an appropriate organizational culture had a significant impact on the success of telemedicine systems in investigated hospitals.

Continuous or in-service training program was also one of the priorities for implementation of the TTCC. The 
topic of education has two aspects in TTCC. First, using the telemedicine system in accordance with the studies carried out in this realm requires acquisition of specific skills for correct and optimal use of this system. In other words, until the personnel do not receive the necessary training in this field, the use of this system will not be possible practically. Second, this system can be applied to continue medical education for doctors, professionals, and other users through seminars, workshops, and distance learning (Ekeland et al., 2010).

One of the success factors of telemedicine is having a formal training program to inform users about actions and technologies of telesurgery (Blignault and Kennedy, 1999). Especially caregivers (specialist doctors) should be well educated about strengths of telemedicine. Considering the significance of teaching staff in the telemedicine system and the fact that telemedicine is heavily dependent on the acceptance of doctors, the importance of providing them with education is twofold (Kifle et al., 2008).

Eadie et al., (2003) and colleagues believe that telemedicine has a great potential to change in various surgeries, but further enhancement and abilities are needed in the field of telesurgery through tactile feedback, instrumentation, telecommunication speed, and availability. Satcher et al., (2014) recommended that health policy-makers, in collaboration with medical experts and regulatory experts, should propose the necessary plans and strategies for the worldwide availability of resources, including technology (telemedicine and telesurgery) to advance cancer care.

In conclusion, in case of the present study, despite various organizational and cultural elements and the support of the Board of Directors and physicians for the implementation of telemedicine in the hospitals, the under study hospitals are currently unable to provide telemedicine services due to various obstacles including the lack of video conferencing equipment, lack of high-speed Internet access for consultation, and inadequate funding for the purchase and implementation of systems for the implementation of telemedicine.

In general, it is necessary to say that to use telemedicine technology on the macro-level of the country, in addition to examining the infrastructure, the funds needed to purchase required equipment must be provided and high-tech special equipment must be implemented in order to enhance the awareness of users. Finally, considering the importance of telemedicine technology and also the development and improvement of the health system development plan in many hospitals of the country, it is recommended to pay special attention to the infrastructure necessary for the development of new technologies, especially telemedicine, along with the advancement of the objectives of clinical and therapeutic priorities in the hospital.

Given the high mortality rate caused by cancers in the country (more than 40,000 cases a year), it is necessary for our country to follow the global advances in preventing and treating various types of cancers. Therefore, the executive culture in this field should be promoted like other infrastructures required for the TTCC program. Based on the results, implementation of TTCC technology in hospitals and other medical centers of the country is successful in effective treatment of various diseases such as cancers. By adopting the views of medical professionals and promoting the organizational and national culture, we can create the successful acceptance of change and utilization of this technology.

\section{Acknowledgments}

The authors want to express a special recognition to the participants who have participated in the data collection of this study, without whom the study could not be possible.

\section{References}

Adami H-O, Hunter DJ, Trichopoulos D (2008). 2nd Edit. Textbook of cancer epidemiology, Monographs in Epidemiology. New York. OXFORD Uni Press.pp, 4.

Bangert D, Doktor R (2000). Implementing store-and-forward telemedicine: Organizational issues. Telemed J E Health, 6, 355-60.

Bangert D, Doktor R (2003). The role of organizational culture in the management of clinical e-health systems. Proceedings of the 36th Annual Hawaii International Conference on System Sciences (HICSS'03).

Blignault I, Kennedy C (1999). Training for telemedicine. J Telemed Telecare, 5, 112-4.

Eadie L, Seifalian A, Davidson A (2003). Telemedicine in surgery. Br J Sur, 90, 647-58.

Chiang Y-H (2013). Using a combined AHP and PLS path modelling on blog site evaluation in Taiwan. Comput Human Behav, 29, 1325-33.

Dargahi H, Razavi, M (2005). an investigation about attitude of clinical physicians in the implementation of telemedicine technology in TUMS hospitals 2003-2004. Tehran Uni Med $J$, 63, 99-107.

Edirippulige S, Marasinghe RB, Dissanayake VH, et al (2009). Eight strategies to promote e-health and telemedicine activities in developing countries. Telehealth Develop World, 1, 79-86.

Ekeland AG, Bowes A, Flottorp S (2010). Effectiveness of telemedicine: a systematic review of reviews. Int $J$ Med Inform, 79, 736-71.

Field MJ (1996). Telemedicine: A guide to assessing telecommunications for health care. Washangton DC. National Academies of Sciences Press. pp, 50.

Hazin R, Qaddoumi I (2010). Teleoncology: current and future applications for improving cancer care globally. Lancet Oncol, 11, 204-10.

Hung K, Zhang YT (2003). Implementation of a WAP-based telemedicine system for patient monitoring. IEEE Trans Inf Technol Biomed, 7, 101-7.

Jennett P, Jackson A, Ho K, et al (2005). The essence of telehealth readiness in rural communities: an organizational perspective. Telemed J E Health, 11, 137-45.

Jennett P, Yeo M, Pauls M, et al (2003). Organizational readiness for telemedicine: implications for success and failure. $J$ Telemedicine Telecare, 9, 27-30.

Kifle M, Mbarika VW, Tsuma C, et al (2008). A telemedicine transfer model for Sub-Saharan Africa. Proceedings of the 41st Annual Hawaii International Conference on System Sciences (HICSS 2008). Waikoloa, HI, USA. IEEE.

Kumar S (2008). Telesurgery: An Audit. In 'Telesurgery'. Springer Publisher. pp, 179.

Leung LC, Cao D (2000). On consistency and ranking of alternatives in fuzzy AHP. Eur J Oper Res, 124, 102-13. 
Mahoozi S, Heidari M, Shahbazi S, et al (2017). Influence of Telemedicine and Telesurgery in Cancer Care training about carcinogenic effects of hookah smoking on the awareness, attitude, and performance of women. Asian Pac J Cancer Prev, 18, 1967.

Mastaneh Z, Alipour J, Hayavi Haghighi M (2010). Managing helath care information systems: A practical approach for health care executives. Bandar Abbas. Rasoul Press, pp,17.

Merchant K, Ward M, Mueller K (2015). Hospital views of factors affecting telemedicine use. Rural Policy Brief, 5,1-4.

Najafi SM, Simforoosh N, Bahl GM, et al (2007). Real-time point to point wireless intranet connection: first implication for surgical demonstration, decision making and telementoring in laparoscopy in Iran. J Birjand Uni Med Sci, 14, 60-6.

Oh H, Rizo C, Enkin M, et al (2005). What is e-Health: a systematic review of published definitions. $J$ Med Internet Res, 7, 32-40.

Pande RU, Patel Y, Powers CJ, et al (2003). The telecommunication revolution in the medical field: present applications and future perspective. Current Surg, 60, 636-40.

Patterson V (2005). Understanding health communication technologies. $J$ Telemedicine Telecare, 11, 54.

ProkoschH-U, Ganslandt T, Dumitru RC, etal(2006). Telemedicine and collaborative health information systems (Telemedizin und Kollaborative Gesundheitsinformationssysteme). IT-Information Technol, 48, 12-23.

Raeissi P, Sharifi M, Khosravizadeh O, et al (2017). Survey of cancer patient safety culture: A comparison of chemotherapy and oncology departments of teaching hospitals of Tehran. Asian Pac J Cancer Prev, 18, 2775-9.

Rahim Zadeh E, Rahim Zadeh S, Azadi S, et al (2013). Feasibility of implementation and deployment telemedicine in Imam Khomeini hospital in Ardabil city. Sci Quarterly Newsletter $J$ Kadoose, 2, 1-11.

Rho MJ, Kim HS, Chung K, et al (2015). Factors influencing the acceptance of telemedicine for diabetes management. Cluster Comput, 18, 321-31.

Satcher RL, Bogler O, Hyle L, et al (2014). Telemedicine and telesurgery in cancer care: inaugural conference at MD Anderson Cancer Center. J Surg Oncol, 110, 353-9.

Shahbazi S, Heidari M (2014). Assessment of the knowledge and attitudes of nursing and midwifery personnel about Breast Self-Examination. Iran J Obstet Gynecol Infertil, 17, 7-12.

Shahbazi S, Heidari M, Ghafourifard M (2017). Comparison of direct and indirect methods of teaching breast self-examination-influence on knowledge and attitudes of Iranian nursing and midwifery personnel. Asian Pac $J$ Cancer Prev, 18, 1157-62.

Stanberry B (2000). Telemedicine: barriers and opportunities in the 21 st century. J Intern Med, 247, 615-28.

Weinstein RS, Lopez AM, Joseph BA, et al (2014). Telemedicine, telehealth, and mobile health applications that work: opportunities and barriers. Am J Med, 127, 183-7.

Wysocki WM, Komorowski AL, Aapro MS (2005). The new dimension of oncology: Teleoncology ante portas. Crit Rev Oncol Hematol, 53, 95-100.

Youlden DR, Cramb SM, Dunn NA, et al (2012). The descriptive epidemiology of female breast cancer: an international comparison of screening, incidence, survival and mortality. Cancer Epidemiol, 36, 237-48.

\section{(ब) $(\mathbb{8}$}

This work is licensed under a Creative Commons AttributionNon Commercial 4.0 International License. 